\title{
Pilot Implementation of a Wellness and Tobacco Cessation Curriculum in North Carolina Group Homes
}

\author{
Hannah M. Baker ${ }^{1} \cdot$ Leah M. Ranney ${ }^{1}$ Adam O. Goldstein ${ }^{1}$
}

Received: 12 September 2014/ Accepted: 8 December 2015/Published online: 28 December 2015

\begin{abstract}
Despite a steady decline in smoking rates in recent decades, individuals with mental illness continue to smoke at disproportionately higher rates than the general population. Adults with mental illness are motivated to quit and quit with rates similar to the general population when evidence-based cessation interventions are used. To build an evidence base for a wellness and cessation curriculum aimed at individuals with mental illness, the Breathe Easy Live Well (BELW) program was pilot tested in two group homes in North Carolina in the spring of 2014. Evaluators conducted pre- and post-implementation site visits and interviews with program instructors to assess outcomes as well as barriers and facilitators to implementation. Qualitative analysis of the data indicated that implementation was successful in both group homes, and the following themes emerged: (1) Training and technical assistance provided throughout implementation was sufficient; (2) Instructors used prior professional experiences and goal setting to facilitate program success and participant engagement; (3) Fostering positive coping strategies contributed to reports of reduced smoking; (4) Curriculum length may be a barrier to recruitment. Additional results included an increased interest among group home residents in more diligently managing mental illness symptoms and
\end{abstract}

\author{
Hannah M. Baker \\ hmbaker2@unc.edu \\ Leah M. Ranney \\ leah_ranney@unc.edu \\ Adam O. Goldstein \\ aog@med.unc.edu \\ 1 Department of Family Medicine, University of North \\ Carolina at Chapel Hill, 590 Manning Drive, CB\#7595, \\ Chapel Hill, NC 27599, USA
}

one group home moving the designated smoking area out of the direct path of the entrance/exit. Results of this pilot project suggest that BELW could be a potentially useful tool for group home staff to address health and wellness along with smoking cessation among individuals with mental illness.

Keywords Tobacco $\cdot$ Mental illness - Cessation · Qualitative analysis · Group home

\section{Introduction}

It has long been recognized that individuals with mental illness smoke at disproportionately higher rates than individuals without mental illness (Lasser et al. 2000; Ziedonis et al. 2008; CDC 2013). Tobacco use in the United States has declined in previous decades, though these reductions are not reflected in adults with mental illness, which have a smoking prevalence between 34 and $88 \%$, compared to $18.3 \%$ in the general population (Ziedonis et al. 2003; Centers for Disease Control and Prevention 2013). This significant disparity may persist in part due to the difficulty in changing tobacco-related norms and an enduring reluctance by mental health providers to address tobacco use among their clients (Johnson et al. 2009; Williams 2008; Morris et al. 2009; Ziedonis et al. 2003; Hitsman et al. 2009). The consensus among many providers has been that tobacco produces a calming and therapeutic effect for patients with mental illness; some staff and patients may believe that smoking alleviates stress or side effects from medication (Campion et al. 2008). Staff may also mistake symptoms of withdrawal from nicotine as increased symptoms of mental illness once patients attempt to quit, thereby furthering the idea that cigarettes produce a 
calming effect in patients (Campion et al. 2008). Some mental health treatment programs may even use cigarettes as an incentive or to reward patients for good behavior (Prochaska 2011; Fagerstrom and Aubin 2009). In addition to this, other institutional barriers persist. For example, when the Joint Commission on the Accreditation of Health Care Organizations proposed a national ban on tobacco use in hospitals, opposition from patient advocacy groups and others resulted in an exemption for psychiatric and drug treatment hospitals (Prochaska 2011).

Despite these barriers, research shows that adults with mental illness are motivated to quit and they quit with rates similar to the general population when evidence-based cessation interventions are used (Lasser et al. 2000; Siru et al. 2009; El-Guebaly et al. 2002). This study details the results of a pilot project that aimed to test the feasibility of implementing the Breathe Easy Live Well (BELW) program in group home settings in North Carolina. BELW is a wellness and cessation curriculum for individuals with mental illness and substance use disorders that has been successfully implemented in other mental health treatment settings (Lee et al. 2011). This suggests that BELW is a promising strategy for addressing health, wellness, and tobacco use among individuals with mental health disorders, and for starting conversations about behavioral change among group home residents. Through evaluating implementation of this curriculum, we sought to explore facilitators and barriers to implementation, as well as the viability of migrating the curriculum to different treatment settings.

\section{Methods}

\section{Pre-implementation}

In January 2014, the North Carolina Southern Regional Area Health Education Center (SR-AHEC) provided a one and a half-day training for instructors implementing BELW. Instructors from each group home were self-selected; after receiving an explanation of the program from SR-AHEC staff, those who were most interested in the program chose to participate as instructor. Group homes also received a monetary incentive for participation in the program from SR-AHEC. Upon completion of the training, evaluators conducted site visits and interviews with three instructors at their respective group homes. Group homes were licensed residential treatment facilities, located in traditional residential neighborhoods, and provided housing and a supported living environment for as many as six residents. Each group home was staffed $24 \mathrm{~h}$ per day by a trained professional. Interviews captured general information about norms, policies, and resident behaviors regarding health and wellness at the group home, for example how often residents exercise, how many residents smoke, etc. Using an environmental checklist, evaluators collected data during site visits describing environmental attributes that may contribute to smoking or wellness-related behaviors. The environmental checklist included attributes such as presence and number of no-smoking signs; exercise equipment available for resident use; number and location of designated smoking areas; presence of vending machines and types of snacks dispensed; and presence of walking paths around the group home, among others. By assessing specific environmental attributes of each group home, evaluation staff were then able to provide evidence for environmental changes that occurred post-implementation, with follow-up questions in the postimplementation guide that elicited if the changes were a result of implementing the curriculum. This was particularly true for the number and location of smoking areas, as well as the presence and number of no-smoking signs. According to North Carolina Statute § 131D-4.4, North Carolina group homes are not allowed to permit smoking inside the residence, and they also must "Conspicuously post signs clearly stating that smoking is prohibited inside the facility." Including this in the checklist helped us to ensure that policies related to smoking were being followed, which was also an indication of the environment each group home cultivated related to smoking and wellness behaviors. Evaluation staff walked around inside as well as outside of the homes, taking notes according to the observational checklist. The overall purpose of the preimplementation site visit was to introduce the evaluation staff to the instructors, to give the evaluation staff a sense of the group home environment, and to ensure the instructors' comfort with reporting their experiences of implementing the program to evaluation staff. Data from the pre-implementation interviews were not transcribed or used in any analysis apart from comparing the environmental checklist from pre- to post-implementation.

\section{5-Week Breathe Easy Live Well Curriculum}

Breathe easy live well is a 15-week curriculum designed to address health and wellness, along with promoting smoking cessation, among individuals with mental illness and/or substance abuse disorders who are in the treatment setting. Utilizing principles from the Stages of Change Model as well as Motivational Interviewing techniques, instructors emphasize self-efficacy and physical well-being, while allowing participants to choose a behavior they would like to work towards improving, all while being a part of a supportive group composed of fellow residents. The chosen behavior does not have to be smoking cessation, but rather could include things like dietary intake, stress reduction, or 
physical activity. By including other behaviors apart from smoking, the curriculum allows for participation from individuals who do not smoke, as well as participation from those who do smoke but are in the pre-contemplation stage of change. By including all individuals, BELW helps to contribute to an inclusive environment in which positive health changes are encouraged and supported among all residents. The primary educational tool is a workbook, which is used by instructors and residents to guide the participants through exercises for each of the fifteen sessions. Exercises are meant to engage participants, and each week emphasizes a different wellness-related topic. Throughout implementation, SR-AHEC staff would periodically call instructors and provide technical assistance as necessary. Residents of the group home voluntarily participate in each week's session. If residents preferred not to participate, they were allowed to do so.

\section{Post-implementation}

Following completion of the 15-week BELW curriculum, evaluators conducted in-depth interviews with two group home staff members and completed two site visits. Interviews lasted no longer than $90 \mathrm{~min}$ and included questions to gather details related to the instructor's experience with running the group. Questions also elicited information about resident participation and interest in the material and subject matter, as well as changes perceived by the instructors in attitudes and/or norms surrounding wellness and smoking behaviors among group home residents. Lastly, interviews elicited information about the usefulness of the initial instructor training as well as usefulness of technical assistance provided throughout implementation by SR-AHEC staff. The interviewer used an interview guide to direct the discussion, which included open-ended questions regarding the above-mentioned topics and then used probes to evoke more in-depth explanation and discussion. Post-implementation interviews were digitally recorded and transcribed using an independent professional service.

\section{Data Analysis}

Following completion of post-interviews and site visits, evaluators developed a codebook consisting of topical codes related to facilitators, barriers, and program implementation. One evaluation staff member coded the data using Atlas.ti qualitative data analysis software (ATLAS.ti Scientific Software Development GmbH, Hardenbergstr. 7, Berlin, Germany), which helped to identify emergent themes related to implementation. Emergent themes were discussed with a second evaluation staff member and themes were prioritized based on the preponderance of data for each theme. Observational data collected from site visits were analyzed by comparing the pre- and post-checklists side-by-side to identify any changes or discrepancies between observed environmental attributes.

\section{Results}

Two of the three group homes participating in the training completed implementation of the full 15-week program. The third group home instructor implemented the first few weeks and then terminated involvement in the program, and she declined post-interview and site visit. Though it is unknown for certain why this instructor terminated involvement in the program, based on information provided to evaluators during pre-implementation interviews, it is possible that this instructor experienced low interest among group home residents, or felt that 15 weeks was too long of a commitment for this particular group home. Program instructors that did complete the 15 -week curriculum reported full participation from all group home residents and reported positive outcomes resulting from the BELW program among program participants. Participants gained interest in becoming healthier overall, including eating healthier and more rigorously managing their symptoms of mental illness.

...when we get together they would ask for grapes and cantaloupe, apples, apple slices or whatever...they were just so much more aware of being healthy so much so that the pharmacy was late delivering meds for one of the individuals, he was like, I can't get sick, I am trying to keep myself together. So, I think that had a lot to do with it because we just have weekly discussion about recovery, smoking, mental illness, substance abuse, whatever it is. (Instructor B)

Instructors noted that most group home residents who smoked began to think about attempting to quit, though most were not ready to quit at the time of program completion. Post-implementation site visits provided observational data that supported evidence of positive outcomes related to the BELW program. One group home moved the designated smoking area farther away from the house so that it was no longer in the direct path of the entrance/exit. Another group home eliminated one smoking area from in front of the home, therefore reducing the total number of smoking areas at the house from three to two. In addition to these outcomes, the following four themes were identified from the interviews. 


\section{Training and Technical Assistance was Sufficient}

Each instructor reported the level of training and technical assistance received was the right amount for successful implementation, despite being less technical assistance that was reported as necessary during previous implementation of the curriculum (Lee et al. 2011). Each instructor also indicated that the guidebooks were their most useful resource.

I didn't need any more assistance at all. We had all our assistance and the book that we had was selfexplanatory. It was very well-written. (Instructor A) I knew that she was always available... but I never got stuck. I think if I got stuck on something, I probably would have reached out to her, but there was never a time when I felt like I needed any additional support. (Instructor B)

\section{Instructors used Prior Experiences and Goal Setting to Facilitate Program Success and Participant Engagement}

Both instructors had extensive experience working with individuals with mental illness and substance use disorders, which helped to facilitate the success of the program in the group home setting. For example, while the curriculum includes quite a bit of interactive, group-oriented material, the instructors at times found it useful to incorporate even more periods of engagement for participants. The curriculum is designed in such a way that it is flexible for instructors to amend it according to their group's participation levels and ability to maintain attention. Using previous experience, Instructor B found that setting goals each week helped to keep participants on track and provided a cohesive way for the program to move forward on a weekto-week basis:

I just think that the things that were most effective for them were consistency because everybody here suffers with a severe and persistent mental illness. So, from time to time, we may have to backtrack, review or whatever, but I think that doing that every week, consistently reminding them of their goals or they are reminding me or updated, you know, let me know if they remembered of their goals. (Instructor B)

Later in the discussion, Instructor B indicated that her experience in the field was very useful to her throughout implementation, and that there was nothing else SR-AHEC could have done to assist her in implementing BELW:

I don't think that there is anything. Being a QP, [Qualified Professional] my primary responsibility is health and safety. So, I have been in the field for 30 plus years. So, I don't know that there is anything that they probably could have or maybe taught me... (Instructor B)

Similarly, Instructor A specifically attributed her experience with nursing and working with individuals with mental illness as helpful throughout instruction:

I used my nursing experience and...my life experience and also my knowledge of dealing with people with mental illness... we took frequent breaks and did more little fun stuff in between the lessons to break up the monotony of just having them sit there and read...So, I broke it up a little bit with everybody participating, making sure that I give everybody a chance to actually read the lines as we do, have their own opinions. (Instructor A)

\section{Fostering Positive Coping Strategies Contributed to Reports of Reduced Smoking}

Instructors indicated that they were able to use the program as a platform for discussions on implementing positive mental health coping strategies, which in turn led to some residents reporting to instructors that they reduced their smoking, or attempted to reduce their smoking. Some residents accomplished this by turning their attention to other tasks throughout the day to reduce cravings; some residents began to provide assistance during meal preparation, while other residents began work on a model train set. One resident re-invigorated efforts to earn his GED, and another decided to fix a bike that was sitting broken in the basement. Importantly, one group home emphasized de-cluttering and organizing of the living space, as well as playing calming music in the evenings as a way to provide a tranquil environment for residents as a coping strategy for managing their mental illness.

So, rather than be out there sitting outside with nothing to do from 12-5, they would be involved in the lunch prep for 12 o'clock and then later on even at 4 o'clock they started getting involved in the meal prep...because one other things that we talked about too was you doing something else with your hands. You got to do something with your hands, whatever situation that you smoke most day, that's the time that you got to focus. (Instructor B)

\section{Curriculum Length May be a Barrier to Recruitment}

Instructors mentioned that the 15 -week program was quite long, and one indicated that she would have preferred that 
it be shorter. Although evaluators were unable to interview the third instructor who did not complete the full 15 weeks of the program, during the pre-implementation interview she also indicated that she felt 15 weeks was a large commitment. This instructor's reluctance to implement the full 15 weeks likely contributed to her inability to complete the program within the group home.

Not the client, but the person who is going to teach it, it might discourage them to participate. So, to me, I think maybe it could be shortened...It could be shortened maybe 10 or something like that, so that more people would participate because I think it's a good program. I liked it a lot, but I don't think I need all that time to teach it. (Instructor A)

\section{Discussion}

Smoking remains the leading cause of preventable death and disease in the United States (Centers for Disease Control and Prevention 2013). Despite great strides in reducing the rates of smoking among the general population, individuals with mental illness continue to smoke at disproportionately higher rates than those without mental illness (Lasser et al. 2000; Ziedonis et al. 2008; Centers for Disease Control and Prevention 2013). Recognizing the need for targeted intervention, the BELW program provides a platform for addressing tobacco use among participants in mental health treatment settings. In this pilot of the program in group home settings, participants in the program experienced a variety of positive outcomes. Evaluation through interviews and environmental observations found that training and technical assistance provided by SR-AHEC was sufficient for implementation; this was in part due to the fact that recruited instructors had extensive experience working in populations of adults with mental health and substance use disorders. The evaluation also found that throughout the program, instructors were able to use the curriculum to help their residents foster positive coping strategies, which in turn led to residents reporting that they had reduced their smoking or attempted to reduce their smoking by engaging themselves in other activities and incorporating stress-reduction strategies into their daily lives. This is important as it suggests that BELW has the potential to move residents of the group homes through the stages of change spectrum. Finally, instructors indicated the length of the BELW curriculum may discourage participation among staff members who may be interested in implementing a wellness and tobacco cessation curriculum within their group home. The original curriculum, Learning About Healthy Living: Tobacco and You, which was used to develop the BELW curriculum was reduced following an extensive evaluation of the program (Williams et al. 2005; Lee et al. 2009). The current curriculum has the potential to be further modified and should continue to be evaluated in order to identify the optimal length. Environmental changes also occurred-smoking areas were either reduced or moved farther away from the entrance/exit, so as to protect residents who did not smoke from exposure to second-hand smoke. This is important as environmental approaches have the ability to reduce smoking by changing the social norms and attitudes about smoking (Brownson et al. 2006).

We recognize several limitations of our findings. These data are from only two group homes, and therefore the themes may not represent a wider implementation of this curriculum in group home settings. However, our findings are consistent with a rigorous evaluation of the BELW curriculum in nine clubhouses (Lee et al. 2009). Additional limitations include not having participant data regarding their experiences with the program, nor self-reported data from residents regarding tobacco quit attempts or tobacco cessation. Instead we relied on second-hand reports from instructors about resident attempts at reducing smoking.

The BELW program is a promising strategy for addressing tobacco use in mental health treatment facilities, and positive outcomes can result when this program is implemented in the group home setting. Implementers of the program should consider reducing the length of the program for maximum success. They should also consider their level of experience working with individuals with mental illness and/or substance abuse disorders, as previous training and experience working with this population seems to assist in the success of implementation. Only two group homes fully implemented the 15 -week program, and further testing of this curriculum in group home settings would help to elucidate the effectiveness of BELW in reducing smoking among group home residents as well as to identify the optimal length and content. However, results of this pilot project suggest that BELW could be a potentially useful tool for group home staff to address health and wellness along with smoking cessation among individuals with mental illness.

\section{References}

Brownson, R. C., Haire-Joshu, D., \& Luke, D. A. (2006). Shaping the context of health: A review of environmental and policy approaches in the prevention of chronic diseases. Annual Review of Public Health, 27, 341-370.

Campion, J., Checinski, K., Nurse, J., \& McNeill, A. (2008). Smoking by people with mental illness and benefits of smoke-free mental health services. Advances in Psychiatric Treatment, 14(3), $217-228$. 
Centers for Disease Control and Prevention. (2013). Vital signs: Current smoking among adults aged $\geq 18$ years with mental illness-United States, 2009-2011. MMWR, 62, 81-87.

El-Guebaly, N., Cathcart, J., Currie, S., Brown, D., \& Gloster, S. (2002). Smoking cessation approaches for persons with mental illness or addictive disorders. Psychiatric Services (Washington, DC), 53(9), 1166-1170.

Fagerstrom, K., \& Aubin, H. J. (2009). Management of smoking cessation in patients with psychiatric disorders. Current Medical Research and Opinion, 25(2), 511-518.

Hitsman, B., Moss, T. G., Montoya, I. D., \& George, T. P. (2009). Treatment of tobacco dependence in mental health and addictive disorders. Canadian Journal of Psychiatry. Revue Canadienne de Psychiatrie, 54(6), 368-378.

Inspection and Licensing of Facilities. (1999). N.C. Gen. Stat. $\S 131 \mathrm{D}-4.4$.

Johnson, J. L., Malchy, L. A., Ratner, P. A., et al. (2009). Community mental healthcare providers' attitudes and practices related to smoking cessation interventions for people living with severe mental illness. Patient Education and Counseling, 77(2), 289-295.

Lasser, K., Boyd, J. W., Woolhandler, S., Himmelstein, D. U., McCormick, D., \& Bor, D. H. (2000). Smoking and mental illness, a population-based prevalence study. JAMA: The Journal of the American Medical Association, 284(20), 2606-2610.

Lee, J. G., Ranney, L. M., Goldstein, A. O., McCullough, A., FultonSmith, S. M., \& Collins, N. O. (2011). Successful implementation of a wellness and tobacco cessation curriculum in psychosocial rehabilitation clubhouses. BMC Public Health, 11, 702 .

Morris, C. D., Waxmonsky, J. A., May, M. G., \& Giese, A. A. (2009). What do persons with mental illnesses need to quit smoking? Mental health consumer and provider perspectives. Psychiatric Rehabilitation Journal, 32(4), 276-284.

Prochaska, J. J. (2011). Smoking and mental illness-Breaking the link. New England Journal of Medicine, 365(3), 196-198.

Siru, R., Hulse, G. K., \& Tait, R. J. (2009). Assessing motivation to quit smoking in people with mental illness: A review. Addiction (Abingdon, England), 104(5), 719-733.

Williams, J. M. (2008). Eliminating tobacco use in mental health facilities: Patients' rights, public health, and policy issues. JAMA: The Journal of the American Medical Association, 299(5), 571-573.

Williams, J., Ziedonis, D., Speelman, N., Vreeland, B., Zechner, M. R., Rahim, R., \& O'Hea, E. L. (2005). Learning about healthy living: Tobacco and you. New Brunswick, NJ: UMDNJ.

Ziedonis, D., Hitsman, B., Beckham, J. C., et al. (2008). Tobacco use and cessation in psychiatric disorders: National Institute of Mental Health report. Nicotine \& Tobacco Research: Official Journal of the Society for Research on Nicotine and Tobacco, 10(12), 1691-1715.

Ziedonis, D., Williams, J. M., \& Smelson, D. (2003). Serious mental illness and tobacco addiction: A model program to address this common but neglected issue. The American Journal of the Medical Sciences, 326(4), 223-230. 